\title{
A patient with metastatic non-small cell lung cancer who received pembrolizumab monotherapy after stereotactic body radiotherapy had progression-free survival of nearly 5 years: a case report
}

\author{
Junjun $\mathrm{Ni}^{1,2 \#}$, Liangwei Yang ${ }^{1,2 \#}$, Huangkai $\mathrm{Zhu}^{3}$, Minghui Chu ${ }^{3}$, Chenxu Zhang ${ }^{3}$, Weidi Zhao ${ }^{3}$, \\ Minglei Yang ${ }^{1,2}$, Xiang Xu ${ }^{1,2}$, Enkuo Zheng ${ }^{1,2}$, Xu Jiang ${ }^{1,2}$, Rui Li ${ }^{1,2}$, Guofang Zhao ${ }^{1,2}$ \\ ${ }^{1}$ Department of Cardiothoracic Surgery, Hwa Mei Hospital, University of Chinese Academy of Sciences (Ningbo No. 2 Hospital), Ningbo, China; \\ ${ }^{2}$ Ningbo Institute of Life and Health Industry, University of Chinese Academy of Sciences (Ningbo No. 2 Hospital), Ningbo, China; ${ }^{3}$ Clinical \\ Department, Medical School of Ningbo University, Ningbo, China \\ \#These authors contributed equally to this work. \\ Correspondence to: Guofang Zhao. Department of Cardiothoracic Surgery, Hwa Mei Hospital, University of Chinese Academy of Sciences (Ningbo \\ No. 2 Hospital), Ningbo, China. Email: guofzhao@hotmail.com.
}

\begin{abstract}
Lung cancer is a malignancy with the highest morbidity and mortality in the world. Radiotherapy, chemotherapy, targeted therapy, and immunotherapy have been widely used to treat metastatic non-small cell lung cancer (NSCLC). Stereotactic body radiotherapy (SBRT), also known as stereotactic ablation radiotherapy (SABR), can precisely deliver a high dose of radiation to a target in a limited area. SBRT has been established as the standard treatment for patients with early NSCLC who are unsuitable for operation or refuse surgery and patients with oligometastatic NSCLC who are not suitable for surgery. As an immunologic agent, pembrolizumab has been approved to treat metastatic NSCLC in certain countries, including China and the United States. Increased tumor proportion score (TPS) can reduce pembrolizumab's immunotherapeutic effect, while SBRT can reduce TPS and enhance immunotherapy efficacy. However, there have been no reports in China on metastatic NSCLC patients who have received pembrolizumab monotherapy after stereotactic body radiotherapy (SBRT). Here, we present a case of progression-free survival (PFS) of nearly 5 years with pembrolizumab monotherapy after SBRT for metastatic NSCLC. This case is the patient with the most prolonged medication duration and who experienced the most efficacious treatment among the patients with metastatic NSCLC reported in the Chinese literature.
\end{abstract}

Keywords: Lung cancer; stereotactic body radiotherapy/stereotactic ablative radiotherapy; anti-programmed death receptor 1; pembrolizumab; case report

Submitted Mar 09, 2021. Accepted for publication Apr 21, 2021.

doi: 10.21037/apm-21-860

View this article at: http://dx.doi.org/10.21037/apm-21-860

\section{Introduction}

Lung cancer is a malignant tumor with the highest morbidity and mortality in the world. The 5 -year survival rates for lung cancer have not improved significantly over the past three decades (1). Stereotactic body radiotherapy (SBRT), also known as stereotactic ablation radiotherapy (SABR), can precisely deliver a high dose of radiation to a target in a limited area. SBRT has been established as the standard treatment for patients with early non-small cell lung cancer (NSCLC) who are unsuitable for operation or refuse surgery and patients with oligometastatic NSCLC who are not suitable for surgery (2). An ongoing clinical trial is being conducted to investigate whether SBRT combined with systemic therapy improves prognosis in patients with oligometastatic tumors, including NSCLC (2-4). The emergence of immune checkpoint inhibitors (ICIs), such as programmed cell death-1 (PD-1)/ programmed death-ligand 1 (PD-L1) antibody, has greatly 
enriched lung cancer treatment modalities. In April 2019, the U.S. Food and Drug Administration (FDA) approved pembrolizumab as a single-agent first-line treatment for patients with a PD-L1 tumor proportion score (TPS) $\geq 1 \%$ and no epidermal growth factor receptor (EGFR)/anaplastic lymphoma kinase (ALK) mutations in stage III or metastatic NSCLC, based on interim results from the 2018 phase III KEYNOTE-042 clinical trial.

Increased TPS can reduce pembrolizumab's immunotherapeutic effect $(3,5)$, while SBRT can reduce TPS and enhance immunotherapy efficacy. Preclinical trials have also shown that $\mathrm{T}$ cell infiltration plays a vital role in post-irradiated tumors, suggesting the potential for synergistic effects of SBRT and immunotherapy. Five-year survival data from the pivotal phase III KEYNOTE-024 trial of pembrolizumab first-line treatment for NSCLC was first announced by Merck et al. at the European Society of Medical Oncology (ESMO) Summit in 2020. The data indicated a 5 -year overall survival (OS) rate in the pembrolizumab group of $31.9 \%$ compared with $16.3 \%$ in the chemotherapy group (6). Here, we present a case of progression-free survival (PFS) of nearly 5 years with pembrolizumab monotherapy after SBRT for metastatic NSCLC. This case is the patient with the most prolonged medication duration and who experienced the most efficacious treatment among the patients with metastatic NSCLC reported in the Chinese literature.

We present the following article in accordance with the CARE reporting checklist (available at http://dx.doi. org/10.21037/apm-21-860).

\section{Case presentation}

A 78-year-old male patient came to Ningbo Hwamei Hospital of the University of Chinese Academy of Sciences for treatment after having multiple nodules found in both lungs during physical examination in a local hospital more than 7 years ago. He had a smoking history of more than 50 years (20 cigarettes/day), chronic cough history of more than 10 years, tuberculosis history, but no family history of lung cancer. His Eastern Cooperative Oncology Group (ECOG) score was 1. Computed tomography (CT) showed that there were mixed density nodules $\left(1,16 \times 19 \times 17 \mathrm{~mm}^{3}\right)$ in the dorsal segment of the lower lobe of the left lung, pure ground-glass nodules (GGOs; 1 in each upper lobe) in both lungs, and aging pulmonary tuberculosis at the apex of both lungs and the dorsal segment of the lower lobe of right lung accompanied by thickening of adjacent pleural adhesions.
Multiple lymph nodes in the left hilum and mediastinum were enlarged, and some of them were accompanied by calcification. To further determine the clinical stage and treatment, he underwent position-emission tomography (PET-CT). PET-CT results showed no metastasis of other organs (Figure 1). According to the results of CT, PET-CT, and other examinations, the nodules in the left lower dorsum lung were considered to be microinvasive or invasive lung cancer (T1N0M0), and the GGO in the right upper lung and the others in the lungs were considered to be inflammatory nodules or carcinoma in situ (multiple primaries). In such cases, we recommend that patients undergo thoracoscopic radical resection of the left lower lung (resection of the dorsal lobe of the left lower lobe and lymph node dissection). After much consideration, the patient and his family decided not to accept surgery or any other treatment.

After 1-year of follow-up, the left lower pulmonary nodules $\left(21 \times 24 \times 21 \mathrm{~mm}^{3}\right)$, and the right upper pulmonary nodules $\left(20 \times 23 \times 21 \mathrm{~mm}^{3}\right)$ were enlarged, while the remaining nodules were stable. The patient's left lower lung and right upper lung nodules were considered multiple primary lung cancer (T1N0M0), and there were still surgical indications. We recommended that the patient receive staging radical resection of the left lower lung nodules and right upper lung nodules. After lengthy consideration, the patient and his family members decided to treat the left lower pulmonary lesion first. SBRT palliative radiotherapy was performed after puncture for definite diagnosis, but combined chemotherapy was refused. The patient received a CT-guided puncture biopsy of the tumor in the left lower dorsal lung, which was pathologically indicated to be cancerous, consistent with lung adenocarcinoma characteristics (due to the small amount of tissue, EGFR, ALK, and other genetic tests could not be performed). The patient then received SBRT palliative radiotherapy for lower left lung cancer in the Radiotherapy Department at a dose of 42.5 Gy/5 F.

After 1 year of SBRT treatment, the patient developed persistent dull pain in the lumbosacral area, which grew progressively worse and was accompanied by radiating pain in the right hip, especially at night, which affected sleep. The patient then returned to the hospital for treatment. Chest high-resolution CT showed significant progression in the lower left pulmonary lesion and slight progression in the upper right pulmonary nodules (Figure 2). PETCT showed that the left lower lung cancer had progressed with left lower lung metastasis, liver metastasis, myocardial 


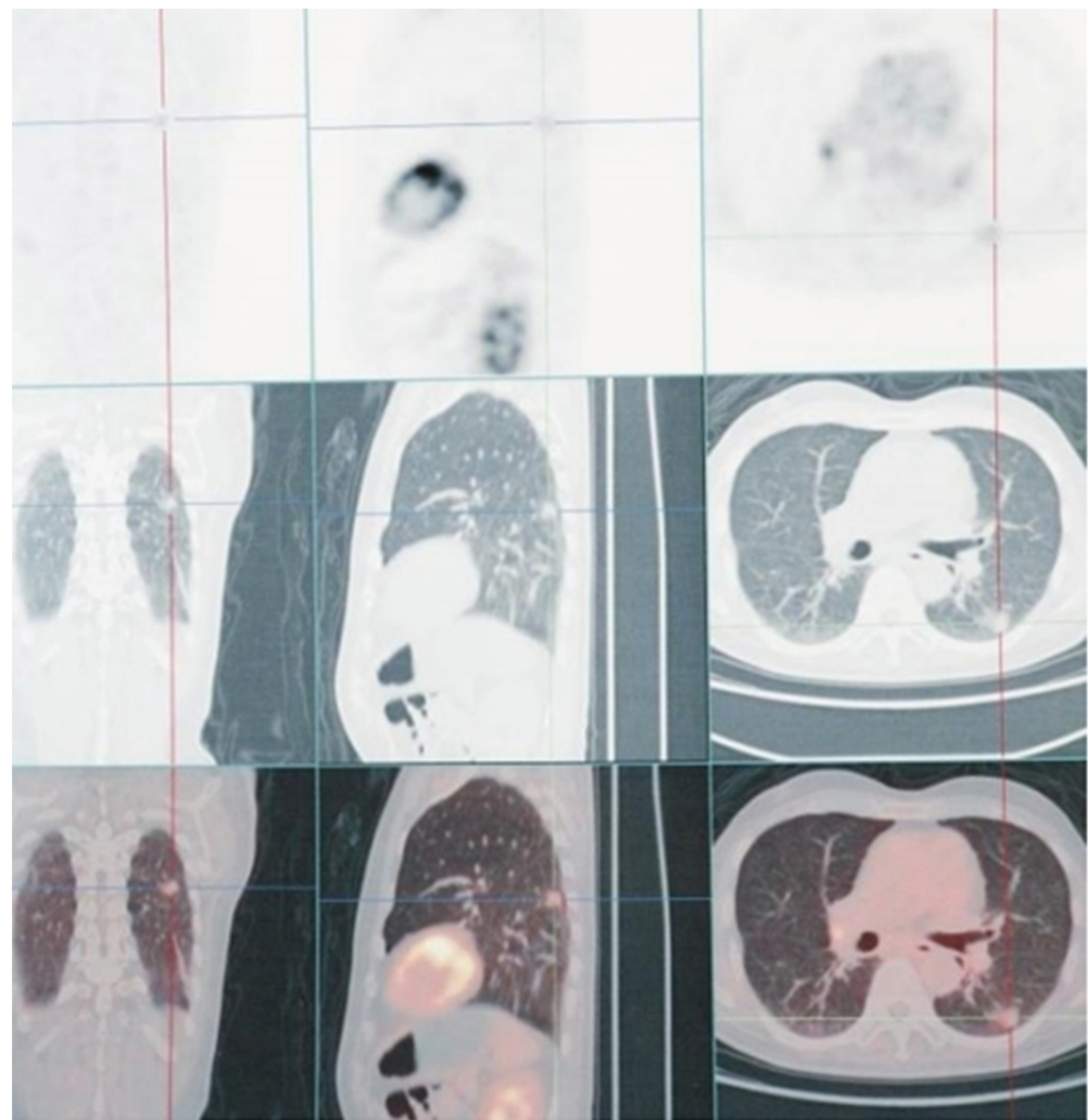

Figure 1 PET-CT images of the patient at the first examination (January 25, 2013). SUVmax 2.63; delayed imaging 2.52. The PET-CT report was consistent with the chest CT report, and no other organ metastasis was observed. PET, position-emission tomography; SUV, standard uptake value; CT, computed tomography.

metastasis, systemic multiple bone metastasis (Figure 3), and severe abnormal liver function, jaundice symptoms, etc. Peripheral blood gene test detected no EGFR and ALK mutations, while BRAF D594G mutation was detected in the circulating tumor (ctDNA), with an abundance of $6 \%$. The patient and his family requested an interdepartmental multidisciplinary team (iMDT) discussion to refine lung cancer treatment and improve prognosis as much as possible.

All procedures performed in studies involving human participants were in accordance with the ethical standards of the institutional and/or national research committee(s) and 

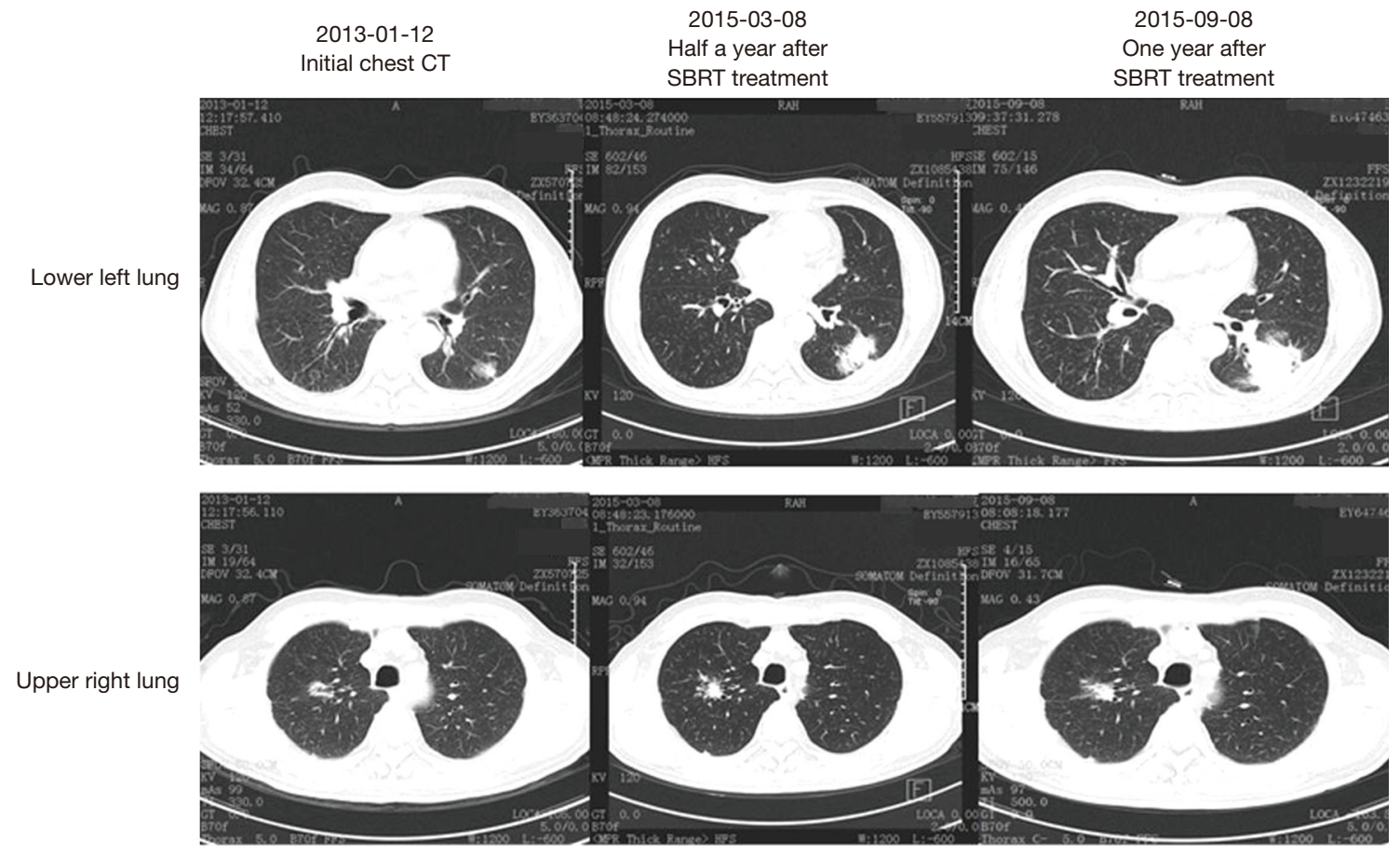

Figure 2 CT images after initial chest CT and SBRT treatment. CT, computed tomography; SBRT, stereotactic body radiotherapy.

with the Helsinki Declaration (as revised in 2013). Written informed consent was obtained from the patient.

\section{iMDT member opinions}

(I) Lu Yang, chief physician of the Radiotherapy Department: SBRT therapy alone is not effective in stopping lung cancer progression, and the patient has progressed to stage IV (T4NxM1). Without intervention, the life expectancy of the patient is approximately 3 months. It is not clear why the patient initially refused the surgery. It is recommended that the patient receive surgical treatment and be treated with SBRT again after surgery.

(II) Suying Qian, chief physician of the HematologyOncology Department: it is suggested that the patient should be treated with the thoracoscopic segmental resection or wedge resection to determine the pathology, and meanwhile, postoperative sacral puncture biopsy to determine the metastatic pathology. According to the pathological results, the patient should be treated with platinum-containing chemotherapy combined with targeted antiangiogenic drugs.
(III) Chuangzhou Rao, chief physician of the Radiotherapy Department: the patient is recommended to undergo sacral puncture biopsy and genetic testing, according to which the next step in the treatment plan can be determined. Based on the KEYNOTE-001 study in October 2015, the FDA approved pembrolizumab for advanced (metastatic) NSCLC cases that progress after other treatments and that are positive for PDL1. Therefore, it is recommended that the patient also receive $\mathrm{PD}-\mathrm{L} 1$ testing to provide a basis for immunotherapy.

(IV) Haibo Shen, chief physician of the Department of Thoracic Surgery: the patient was diagnosed with early-stage lung cancer, which has now progressed to stage IV lung cancer and cannot be treated by radical surgery. SBRT or SBRT combined with targeted antiangiogenesis therapy is recommended.

(V) Guofang Zhao, director of the Department of Thoracic Surgery: the patient did not respond well to SBRT treatment and progressed to stage IV lung cancer (T4NxM1). Without intervention, the life expectancy is about 3 months. It is recommended that sacral puncture biopsy to identify metastases and platinum-containing chemotherapy combined with 

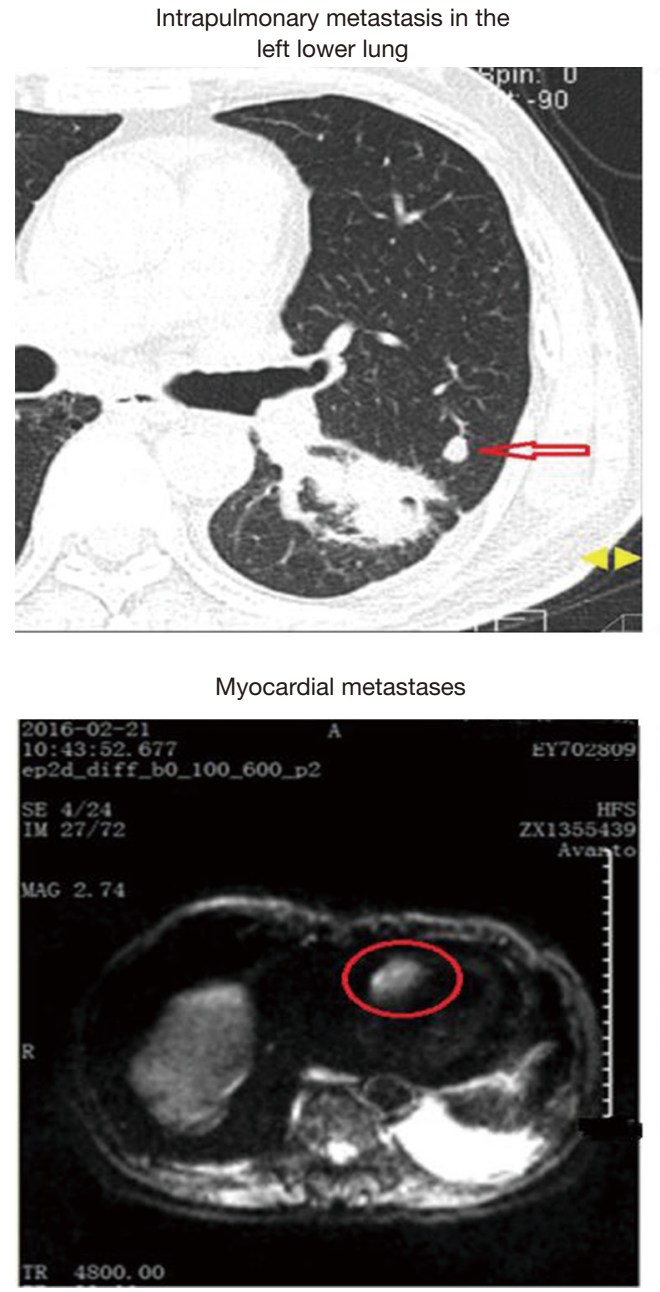

Figure 3 Metastatic lesions. S1, sacral vertebrae 1.

targeted antiangiogenic therapy be considered (but the family of the patient rejects any chemotherapyrelated regimen). Other recommendations include palliative radiotherapy at the sacrum for pain control, zoledronic acid injection anti-bone destruction therapy (once a month), nutritional support therapy, and regular follow-up.

\section{Treatment and follow-up}

The patient underwent a puncture biopsy of sacral metastases, and the pathologic findings indicated sarcomatoid carcinoma. Palliative analgesic radiotherapy was given with the following parameters: radiation field spanning from the L4 to the S3 vertebral body, a planning target volume (PTV) of 95\%, $40 \mathrm{~Gy} / 20 \mathrm{~F}$, and anti-

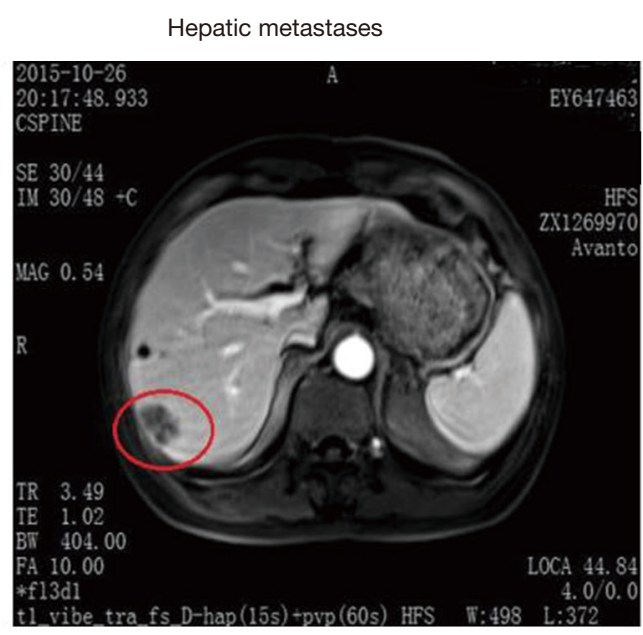

$\mathrm{S} 1$ metastases in the sacrum

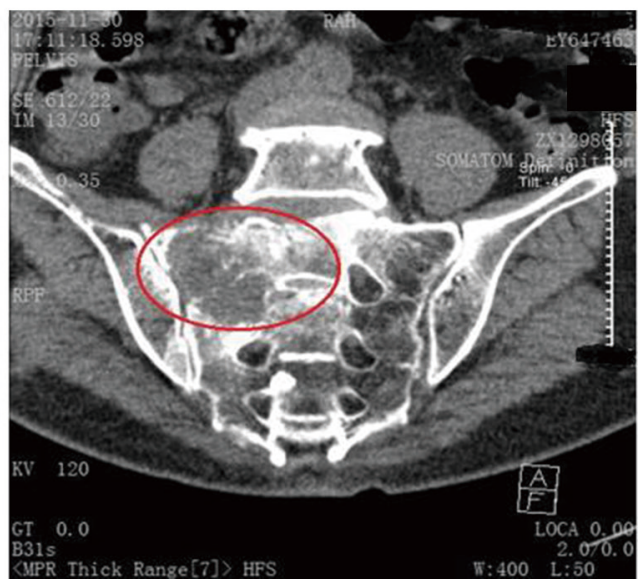

bone destruction treatment with zoledronic acid injection once a month. The above methods were found to have poor efficacy after 3 months of treatment, and the CT reexamination (February 2016) suggested that both the primary and metastatic lesions were progressing rapidly. On February 22, 2016, the patient began to receive pembrolizumab treatment $(200 \mathrm{mg} /$ time, every 21 days as a course of treatment) without PD-L1 expression detection (no formal PD-L1 detection pathway existed in China at that time).

After 4 months of treatment, a follow-up examination found that the patient's cough, shortness of breath, pain, and other symptoms were significantly relieved. CT reexamination (June 28, 2016) indicated that the left lower lung lesion (Figure 4) and metastatic lesions (Figures 5-7) were significantly reduced. It has been more than 7 years 
2016-02-21 Keytruda treatment baseline
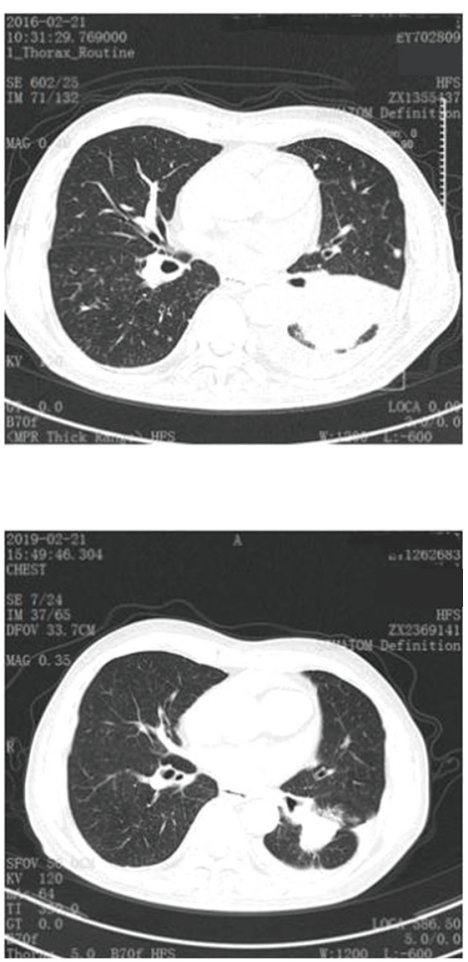

2019-02-21

3 years of keytruda treatment
2016-11-23

9 months of keytruda

treatment
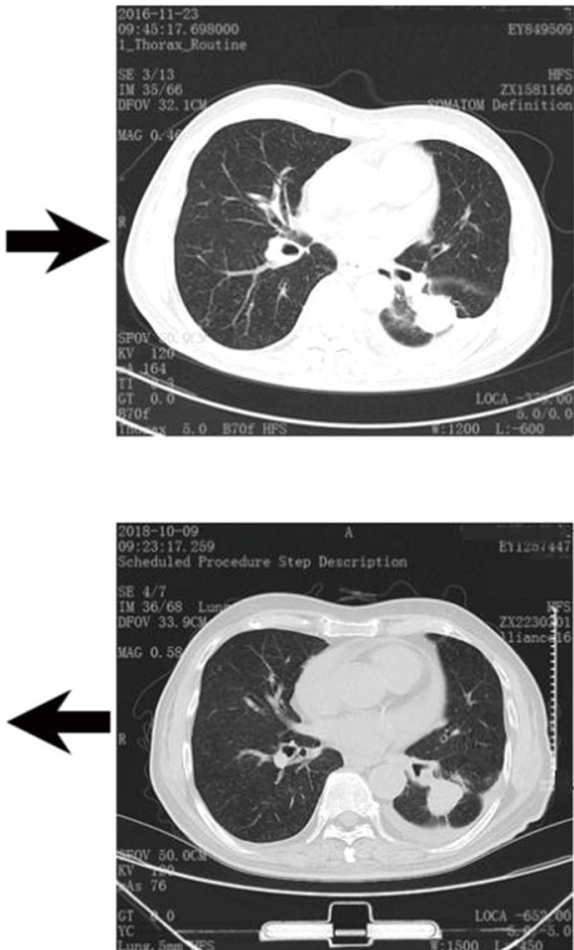

2018-10-09

32 months of keytruda treatment
2017-11-21

21 months of keytruda treatment

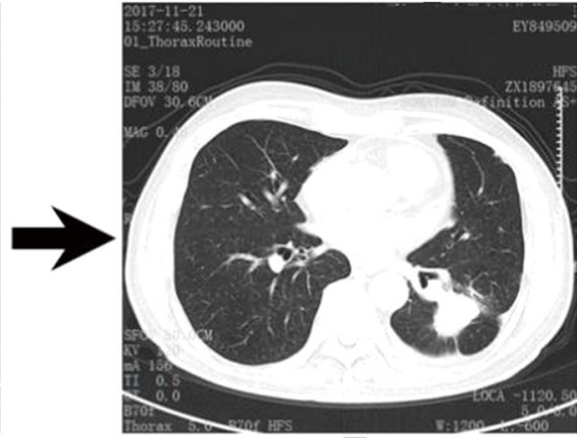

$\forall$

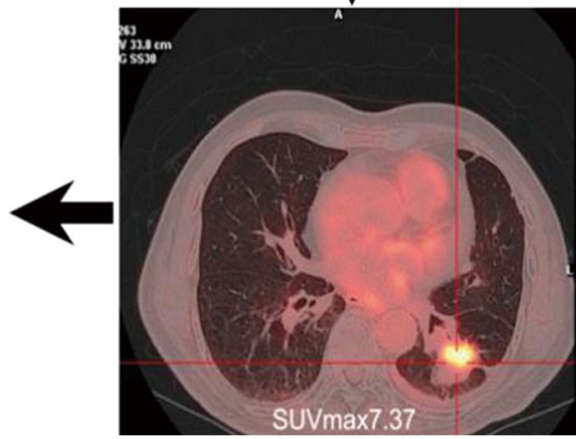

2018-03-07

PET-CT reexamination

Figure 4 Left lower pulmonary lesion after pembrolizumab treatment.

since the patient discovered the lesion. After extensive metastasis, the patient obtained 52 months of clinical benefit and good quality of life after monotherapy, with no noticeable adverse reactions (Figure 8).

\section{Discussion}

Whether SBRT combined with pembrolizumab can increase the toxic reaction of patients has been much discussed.

Since the introduction of ICIs, many studies have been carried out to investigate the changes in immunotoxicity caused by ICIs in combination with other treatments. Shaverdian et al. (7) retrospectively analyzed the data from one of the centers in the KEYNOTE-001 phase 1 trial (University of California, Los Angeles, CA, USA). They found pulmonary toxicity in 15 (63\%) of 24 lung cancer patients treated with pembrolizumab after thoracic RT, 3 of whom (13\%) were associated with treatment, compared with 29 (40\%) of 73 lung cancer patients not treated with thoracic RT, 1 of whom (1\%) was associated with treatment. This appears to show that SBRT increases the response to immunotherapy but does not increase toxicity.

In the prospective randomized controlled clinical trials by Luke et al. (3), multisite SBRT combined with pembrolizumab had good efficacy and low toxicity in treating tumors, including lung cancer. Furthermore, significant differences were found in the expression of interferon-gamma-related genes between tumor biopsies treated with SBRT and those that did not receive irradiation. The toxicity of SBRT combined with pembrolizumab is often present in the irradiated area, making it difficult to distinguish the toxicity of combination therapy from radiotherapy alone. In a retrospective study by Bang et al. (8), 4\% of patients with anti-PD-1 treatment followed by palliative radiotherapy had grade 3 and above immune-related toxicity, which was independent of RT treatment location. Although SBRT does not increase 


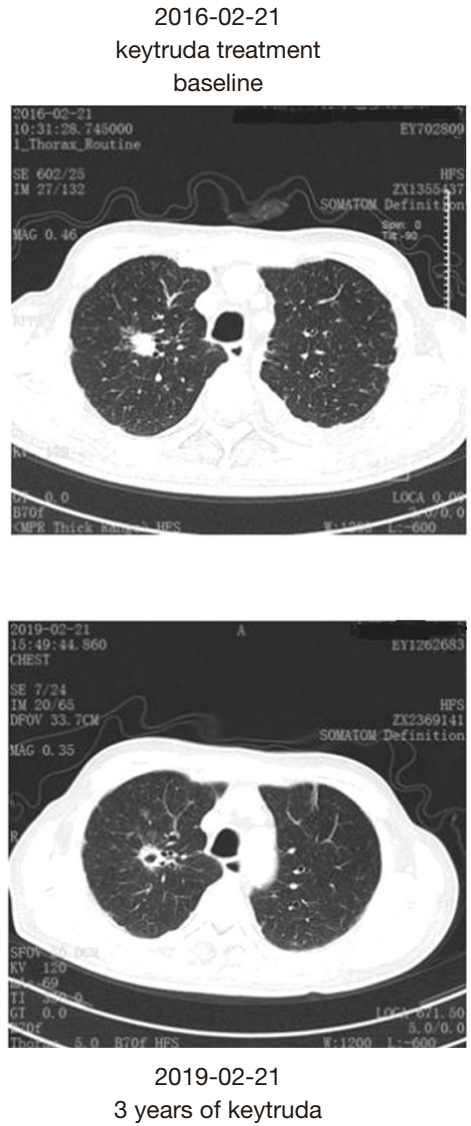

3 years of keytruda treatment
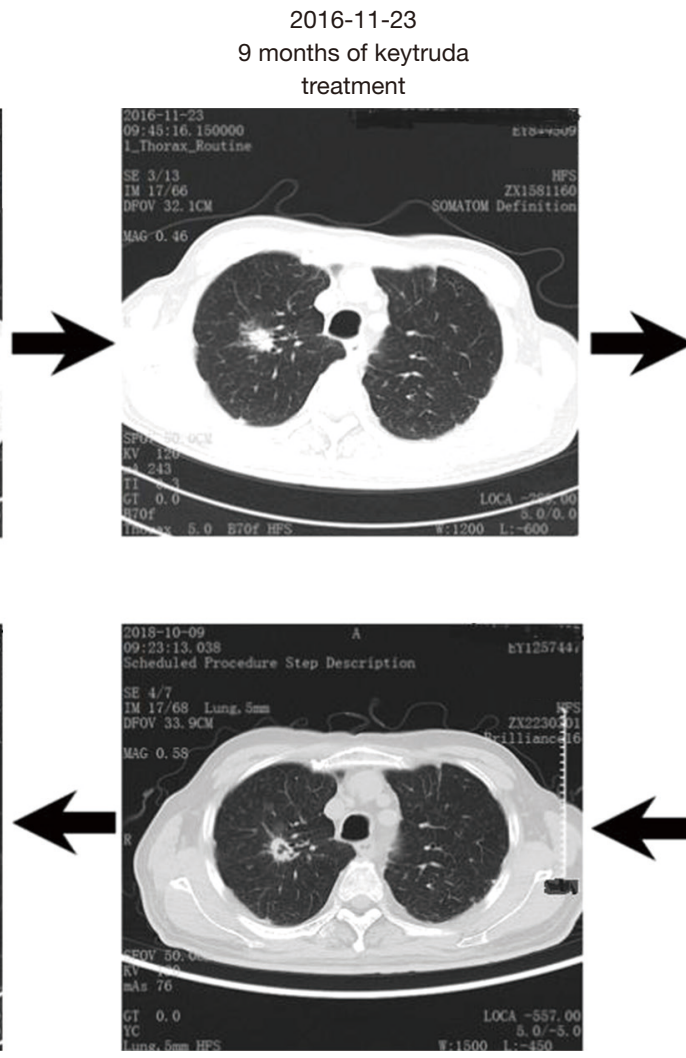

2018-10-09 32 months of keytruda treatment
2017-11-21

21 months of keytruda treatment
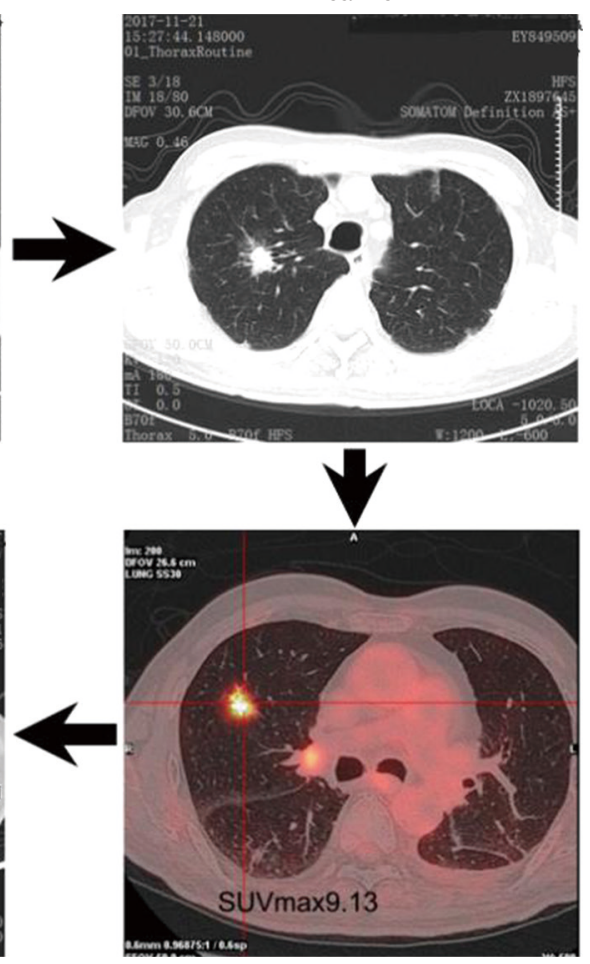

2018-03-07

PET-CT reexamination

Figure 5 Right upper pulmonary lesion after pembrolizumab treatment.

overall toxicity during pembrolizumab therapy, this study seems to suggest that RT increases the incidence of grade 3 and higher immune-related toxicity during anti-PD-1 therapy through systemic responses.

Whether SBRT combined with anti-PD-1 immunotherapy is the best deterministic treatment for stage III or metastatic NSCLC patients who refuse chemotherapy and have negative genetic tests for markers such as EGFR, has also received research attention.

Chicas-Sett et al. (2) retrospectively analyzed the results of 18 studies (including 6 prospective studies) and found that compared with SBRT alone, the ICI-SBRT combination had good safety, higher local control rate, higher absolute response rate, and longer PFS. More data are needed to confirm whether SBRT combined with antiPD-1 immunotherapy is the best deterministic treatment for stage III or metastatic NSCLC lung cancer.

Immunotherapy has changed the treatment model of NSCLC to some extent. Adding other treatments to ICI may be an excellent way to approach optimal treatment (9). RT-ICI combination therapy is a promising approach, and its exact efficacy and toxicity are being investigated. For example, a recent study showed that nanoparticles have the potential to enhance abstract reactions in RT-ICI combinations (10). The ongoing retrospective studies and prospective trials will help us determine whether SBRT combined with anti-PD-1 immunotherapy is the best definitive treatment for stage III or metastatic NSCLC lung cancer and aid in clarifying the appropriate treatment criteria in the future.

Although several studies have shown that combined treatment with SABR and ICI increases median OS and PFS, a pooled analysis of these studies does not indicate a general increase in median OS and PFS. Rather, most studies have shown increased rates of distant/absolute immune response in patients treated with SABR and ICIs, suggesting that radiotherapy has the potential to transform traditional non-responders into responders. Most of the 


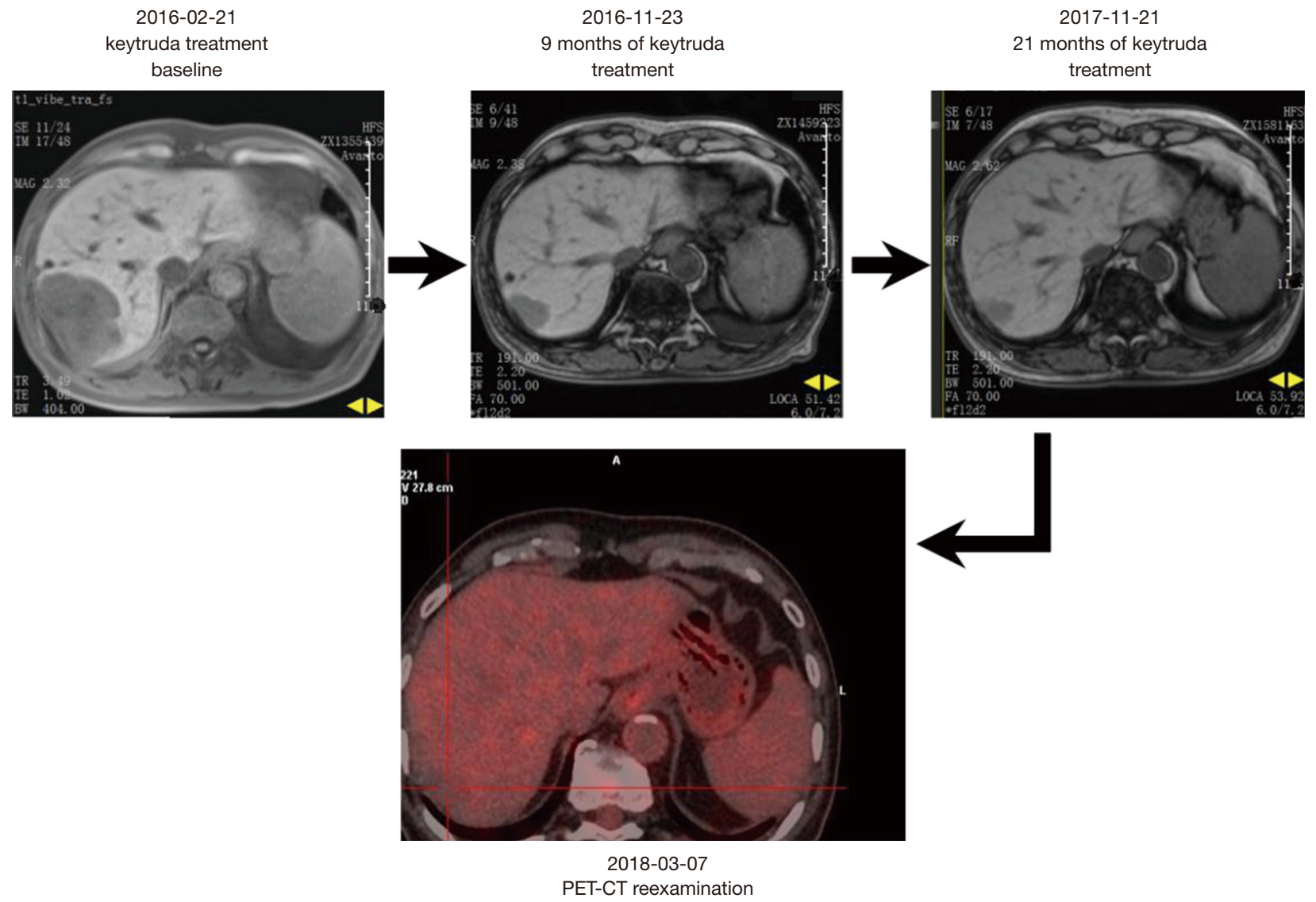

Figure 6 Liver metastases after pembrolizumab treatment.

patients treated with ICIs and radiotherapy in the study were unable to receive further systemic treatment after their disease deteriorated, which may mean that the apparent clinical benefit does not translate into the prolonged OS and PFS. Based on the scientific evidence presented thus far, this treatment combination appears to be the best deterministic treatment for stage III or metastatic NSCL cases who refuse chemotherapy and are negative for genetic markers such as EGFR. Further prospective studies may provide insights into the ideal treatment regimen regarding optimal RT timing, dose, and grading.

\section{Reflection on the case}

In this case, the patient was treated with anti-PD-1 immunotherapy(pembrolizumab) without detection of PD-L1 expression when both the primary and metastatic lesions had progressed significantly, and a relatively ideal therapeutic effect was obtained. In our opinion, this patient is a special case whose treatment course does not necessarily have extensive clinical application value. Nevertheless, should similar situations once again arise, especially in patients with advanced metastatic NSCLC, we should consider the PD-L1 expression test. Anti-PD-1 immunotherapy, including pembrolizumab, for monoimmunotherapy or immuno-combined chemotherapy, is also a more popular and effective treatment option for advanced patients.

\section{Limitations}

Throughout the patient's diagnosis and treatment process, there were two ideal surgical opportunities, but neither the patient nor his family members opted for surgical treatment, which further led to the progression of the disease to stage IV (T4NxM1). This reluctance from the patient's family members stemmed from a fear that the operation would adversely affect the quality of life of the elderly patient, but this was likely exacerbated by poor communication by our doctors. 
A

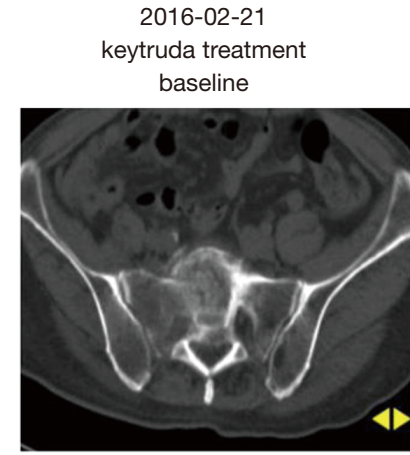

2016-11-23

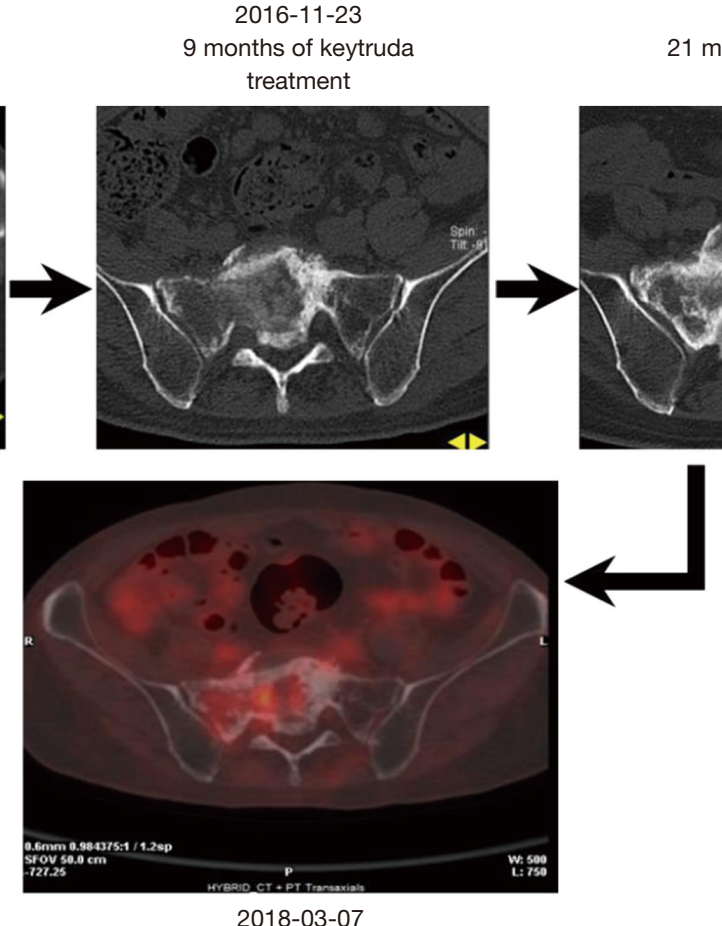

2017-11-21

1 months of keytruda treatment

PET-CT reexamination

B

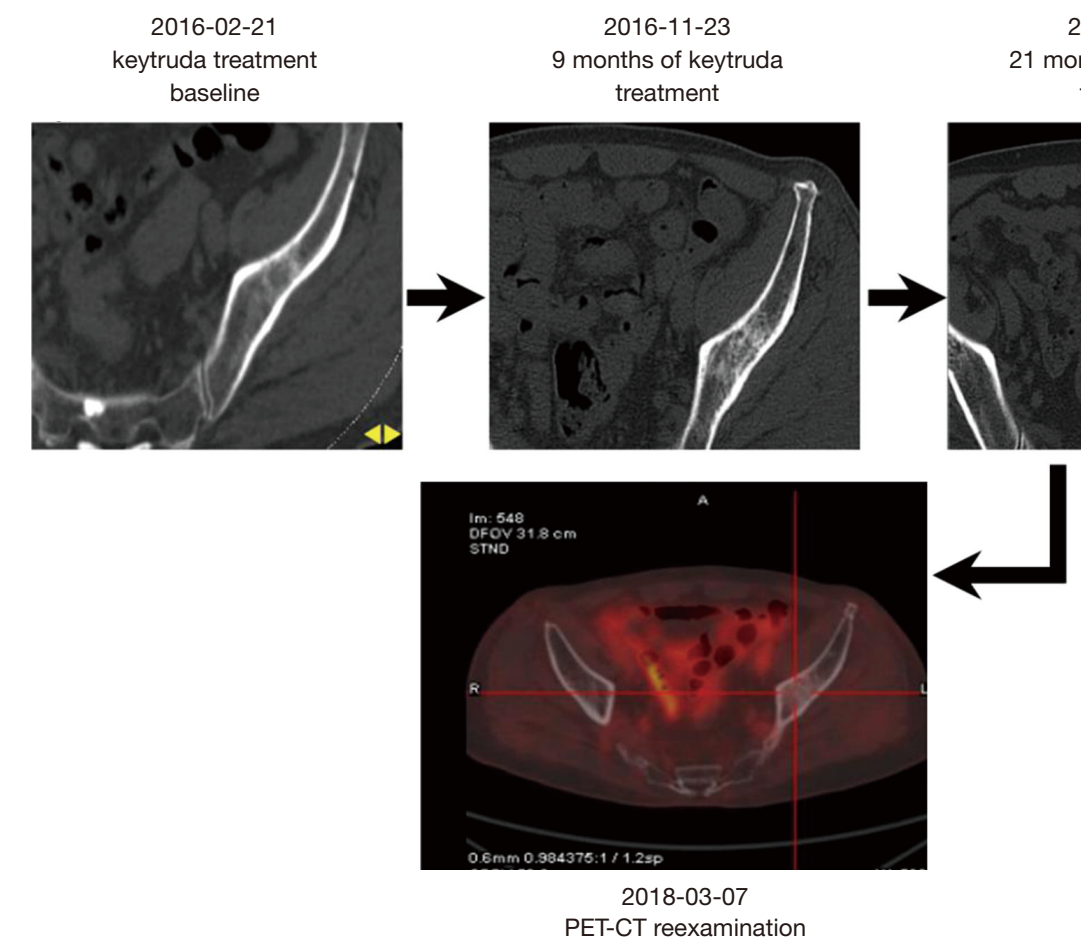

2017-11-21

months of keytruda

treatment

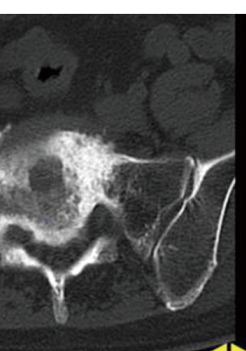

\section{(1)}

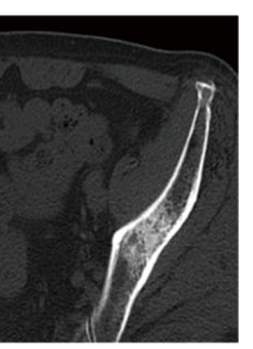

Figure 7 Metastases in the sacroiliac joint after pembrolizumab treatment. (A) Metastases in the sacral S1; (B) metastases in the left iliac crest. PET, position-emission tomography; CT, computed tomography. 


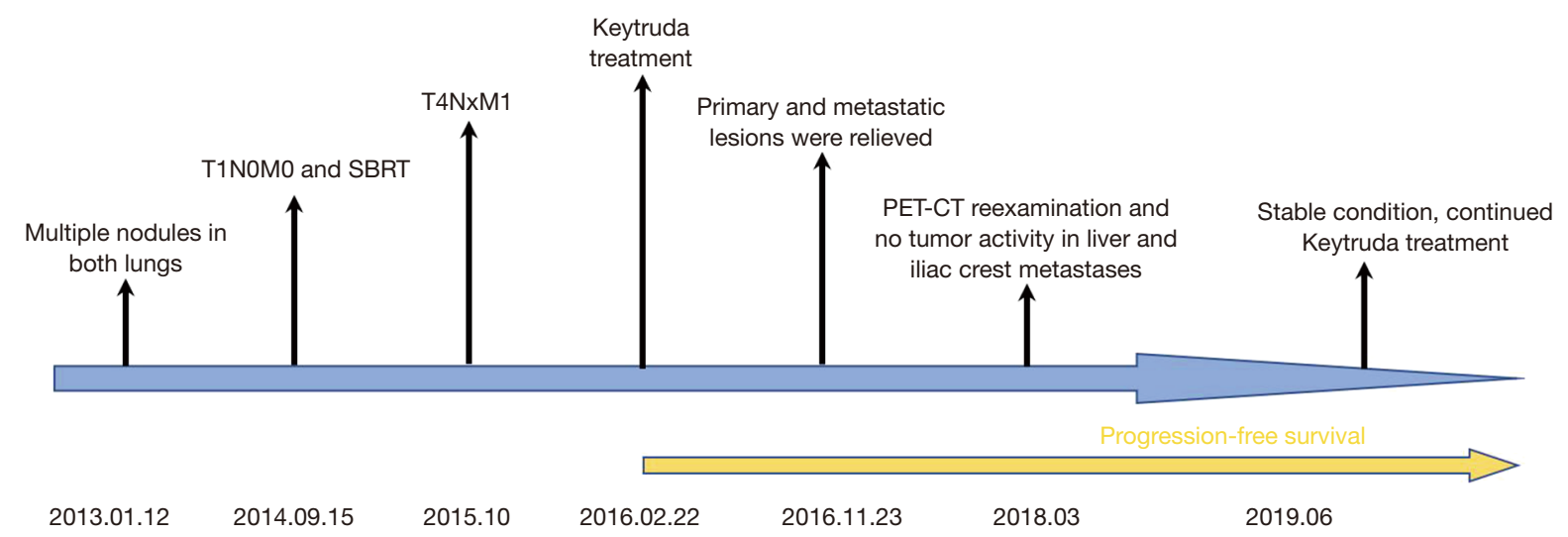

Figure 8 Diagnosis and treatment process. SBRT, stereotactic body radiotherapy; PET-CT, position-emission tomography-computed tomography.

Due to the aggravation that can arise from potential misunderstandings between doctors and patients, communication between doctors and patients has become an essential link in clinical medical practice. Good patient communication is an essential skill in becoming an excellent clinician.

\section{Conclusions}

Since February 2016, this patient has received pembrolizumab monotherapy for 52 months; he has become the patient with the most prolonged medication duration with the best efficacy among Chinese patients with metastatic NSCLC as reported in the literature. Most studies have preliminarily demonstrated that SBRT combined with anti-PD-1 therapy can improve patient outcomes with lower toxicity than SBRT alone or anti-PD-1 monotherapy. Still, more clinical trials are needed to prove its efficacy. This also represents a new research direction in exploring the corresponding treatment criteria, including radiation dose, tumor size, and the time between combined treatment, during SBRT combined with anti-PD-1 treatment.

\section{Acknowledgments}

Funding: This study was supported by Medical Health Science and Technology Project of Zhejiang Provincial Health Commission (Grant No. 2021KY1009) and Key discipline of Hwamei Hospital, University of Chinese Academy of Science (Grant No. 2020ZDXK03).

\section{Footnote}

Reporting Checklist: The authors have completed the CARE reporting checklist. Available at http://dx.doi.org/10.21037/ apm-21-860

Conflicts of Interest: All authors have completed the ICMJE uniform disclosure form (available at http://dx.doi. org/10.21037/apm-21-860). The authors have no conflicts of interest to declare.

Ethical Statement: The authors are accountable for all aspects of the work in ensuring that questions related to the accuracy or integrity of any part of the work are appropriately investigated and resolved. All procedures performed in studies involving human participants were in accordance with the ethical standards of the institutional and/or national research committee(s) and with the Helsinki Declaration (as revised in 2013). Written informed consent was obtained from the patient.

Open Access Statement: This is an Open Access article distributed in accordance with the Creative Commons Attribution-NonCommercial-NoDerivs 4.0 International License (CC BY-NC-ND 4.0), which permits the noncommercial replication and distribution of the article with the strict proviso that no changes or edits are made and the original work is properly cited (including links to both the formal publication through the relevant DOI and the license). See: https://creativecommons.org/licenses/by-nc-nd/4.0/. 


\section{References}

1. Goldstraw P, Chansky K, Crowley J, et al. The IASLC Lung Cancer Staging Project: Proposals for Revision of the TNM Stage Groupings in the Forthcoming (Eighth) Edition of the TNM Classification for Lung Cancer. J Thorac Oncol 2016;11:39-51.

2. Chicas-Sett R, Morales-Orue I, Castilla-Martinez J, et al. Stereotactic Ablative Radiotherapy Combined with Immune Checkpoint Inhibitors Reboots the Immune Response Assisted by Immunotherapy in Metastatic Lung Cancer: A Systematic Review. Int J Mol Sci 2019;20:2173.

3. Luke JJ, Lemons JM, Karrison TG, et al. Safety and Clinical Activity of Pembrolizumab and Multisite Stereotactic Body Radiotherapy in Patients With Advanced Solid Tumors. J Clin Oncol 2018;36:1611-8.

4. Wong AC, Watson SP, Pitroda SP, et al. Clinical and molecular markers of long-term survival after oligometastasis-directed stereotactic body radiotherapy (SBRT). Cancer 2016;122:2242-50.

5. Huang AC, Postow MA, Orlowski RJ, et al. T-cell invigoration to tumour burden ratio associated with antiPD-1 response. Nature 2017;545:60-5.

6. Merck NJ. First-Line Treatment With Merck's KEYTRUDA@ (pembrolizumab) Doubled Five-Year

Cite this article as: Ni J, Yang L, Zhu H, Chu M, Zhang C, Zhao W, Yang M, Xu X, Zheng E, Jiang X, Li R, Zhao G. A patient with metastatic non-small cell lung cancer who received pembrolizumab monotherapy after stereotactic body radiotherapy had progression-free survival of nearly 5 years: a case report. Ann Palliat Med 2021;10(4):4999-5009. doi: 10.21037/apm-21-860
Survival Rate (31.9\%) Versus chemotherapy (16.3\%) in Certain Patients With Metastatic Non-Small Cell Lung Cancer Whose Tumors Express PD-L1 (TPS >50\%). MERCK 2020. Available online: https://www.businesswire. com/news/home/20200921005172/en/

7. Shaverdian N, Lisberg AE, Bornazyan K, et al. Previous radiotherapy and the clinical activity and toxicity of pembrolizumab in the treatment of non-small-cell lung cancer: a secondary analysis of the KEYNOTE-001 phase 1 trial. Lancet Oncol 2017;18:895-903. Erratum in: Lancet Oncol 2017;18:e371.

8. Bang A, Wilhite TJ, Pike LRG, et al. Multicenter Evaluation of the Tolerability of Combined Treatment With PD-1 and CTLA-4 Immune Checkpoint Inhibitors and Palliative Radiation Therapy. Int J Radiat Oncol Biol Phys 2017;98:344-51.

9. Onoi K, Chihara Y, Uchino J, et al. Immune Checkpoint Inhibitors for Lung Cancer Treatment: A Review. J Clin Med 2020;9:1362.

10. Morales-Orue I, Chicas-Sett R, Lara PC. Nanoparticles as a promising method to enhance the abscopal effect in the era of new targeted therapies. Rep Pract Oncol Radiother 2019;24:86-91.

(English Language Editor: J. Gray) 